\title{
CONFECÇÃO E INSTALAÇÃO DE BIODECOMPOSITORES ORGÂNICOS EM ESCOLAS DE EDUCAÇÃO BÁSICA DE GAROPABA - SC
}

\author{
Sabrina Moro Villela Pacheco \\ Instituto Federal de Santa Catarina - Campus \\ Garopaba \\ sabrinap@ifsc.edu.br \\ Rogers Barbi \\ Instituto Federal de Santa Catarina - Campus \\ Garopaba \\ rogers@ifsc.edu.br \\ Charles Veleda Broqua \\ Instituto Federal de Santa Catarina - Campus \\ Garopaba \\ militec.rs@hotmail.com
}

\author{
Noeli Catarina Pazetto \\ EPAGRI \\ noelipazetto@gmail.com
}

Anderson Kangerski

EPAGRI

andersonk@epagri.sc.gov.br

\begin{abstract}
Resumo
Levando-se em consideração a problemática planetária ambiental associada ao descarte incorreto dos resíduos orgânicos, o presente projeto teve como principal objetivo confeccionar e instalar em escolas públicas de Garopaba - SC sete biodecompositores orgânicos no período de abril a agosto de 2013. O dispositivo citado foi idealizado pela Empresa de Pesquisa Agropecuária e Extensão Rural de Santa Catarina e foi construído para o descarte de resíduos orgânicos alimentares gerados pelas cozinhas das escolas. Além da implantação dos biodecompositores, diversas palestras para a conscientização da comunidade escolar foram realizadas com ilustração de experiências lúdicas para facilitar a compreensão dos processos biológicos de decomposição e entrega de cartilhas explicativas. Foi possível atingir um público total de 1000 pessoas, que, por sua vez, deverão auxiliar na divulgação e multiplicação das informações trabalhadas.

Palavras-chave: Biodecompositor. Resíduos orgânicos. Meio ambiente
\end{abstract}

\section{MANUFACTURE AND INSTALLATION OF ORGANIC BIO-DECOMPOSERS IN EDUCATION BASIC SCHOOLS OF GAROPABA - SC}

\begin{abstract}
Taking into account the global environmental problem associated with the incorrect disposal of organic waste, this project aimed to fabricate and install in public schools in Garopaba - SC seven organic bio-decomposers in the period April to August 2013. The apparatus was designed by Agricultural Research and Rural Extension of Santa Catarina quoted and was built for the disposal of organic food waste generated by school kitchens. Besides the implementation of bio-decomposers, several lectures to raise awareness of the school community were carried out with illustration of playful experiences to facilitate the understanding of the biological processes of decomposition and delivery explanatory booklets. It covered a total audience of 1000 people, which in turn, should assist in the dissemination of information and multiplication worked. Keywords: Bio-decomposer. Waste residues. Environment
\end{abstract}




\section{PRODUCCIÓN Y INSTALACIÓN DE BIODECOMPOSITORES ORGÁNICOS EN LAS ESCUELAS PRIMARIAS GAROPABA - SC}

\section{Resumen}

Teniendo en cuenta los problemas ambientales globales asociados a la disposición inadecuada de los residuos orgánicos, este proyecto apunta a fabricar e instalar en las escuelas públicas en Garopaba - SC, siete biodecompositores orgánicos de abril a agosto de 2013. El mencionado dispositivo fue diseñado por la Investigación y Extensión Rural Compañía Agrícola de Santa Catarina y fue construida para la eliminación de residuos de alimentos orgánicos generados por las cocinas escolares. Además de la implementación de biodecompositores, varias conferencias de sensibilización de la comunidad escolar se realizaron con la ilustración de experiencias lúdicas para facilitar la comprensión de los procesos biológicos de la descomposición y la entrega de folletos explicativos. Fue posible llegar a un público total de 1.000 personas, lo que, a su vez, debe ayudar en la difusión y proliferación de la información funcionado.

Palavras clave: Biodecompositor. Residuo orgánico. Ambiente 
Confecção e instalação de biodecompositores orgânicos em escolas de educação básica de Garopaba - SC

\section{INTRODUÇÃO}

O planeta terra está vivenciando o alerta da degradação ambiental que impulsiona a adoção de novas mudanças nos processos produtivos de bens, diminuição do consumo energético e novas posturas em relação aos dogmas tradicionais da economia de mercado. É sabido que o descarte inadequado de resíduos sólidos é responsável por grandes danos ambientais. Os resíduos sólidos podem ser definidos como quaisquer material resultante das atividades diárias do homem (BIDONE; POVINELLI, 1999). Segundo o Atlas de Saneamento do IBGE de 2011, a situação dos resíduos sólidos no Brasil é alarmante, pois a maior parte das cidades brasileiras apresenta sistema de coleta de resíduos, porém, cerca de 50,8\% faz sua destinação para espaços a céu aberto - os chamados lixões (IBGE, 2011). Ainda de acordo com esse mesmo relatório, os pequenos municípios são os que mais se destacam por descartar inadequadamente seus resíduos. Cerca de 50\% dos municípios com até 20 mil habitantes descartam seus resíduos em lixões. O município de Garopaba possui atualmente uma população de 18.138 e, desse modo, seguiu a mesma lógica apresentada até o final dos anos 90, momento em que os resíduos produzidos eram descartados em um lixão localizado na periferia da cidade (IBGE, 2013). Atualmente, os resíduos são enviados ao aterro sanitário localizado no município de Biguaçu. Aproximadamente metade de todo o material enviado ao referido aterro é constituído por resíduos sólidos orgânicos (IPEA, 2012).

Segundo as normas da ABNT, os resíduos sólidos podem ser classificados como pertencentes às Classes I, IIa e IIb. Os resíduos sólidos orgânicos biodegradáveis, tais como restos de alimentos, cascas de frutas, sacos de papel usados para preparação de café, resíduos do corte de grama, poda de arbustos, podem ser classificados na classe IIa (ABNT, 2004). De acordo com esta norma, os resíduos orgânicos podem ser dispostos em aterros sanitários ou reciclados sem oferecer perigo ao meio ambiente. Esta última citação é relativa, pois compara os resíduos orgânicos aos resíduos hospitalares, industriais e outros, significativamente mais danosos ao meio como um todo.

Levando-se em consideração esse contexto e com o objetivo de reduzir a quantidade de resíduos gerados pelo município de Garopaba - SC, a adoção de medidas que visem a reciclagem de tais materiais devem ser implementadas. A instalação de biodecompositores é um dos caminhos a serem adotados para modificar o quadro apresentado. Os eventos que ocorrem nos biodecompositores baseiam-se em processos biológicos de decomposição da matéria orgânica e que possui como produto final um material que pode ser aplicado no solo para melhorar suas 
Confecção e instalação de biodecompositores orgânicos em escolas de educação básica de Garopaba - SC

características físicas, químicas e biológicas. Em outras palavras, esse processo valoriza o resíduo sólido orgânico e traz inúmeros benefícios quando aplicado ao solo pois ajuda na manutenção da umidade do solo, preserva contra a erosão, melhora as propriedades biológicas e aumenta a permeabilidade que favorece o estabelecimento de minhocas e besouros (INÁCIO; MILLER, 2009).

A compostagem pode ocorrer por dois métodos, o método em que o material orgânico é levado para um local e disposto em pilhas de formato variável onde a aceleração necessária para o desenvolvimento do processo de decomposição biológica é atingida por revolvimentos periódicos; e o método acelerado, onde a aeração é forçada por tubulações perfuradas (JARDIM et al., 2000). No caso dos biodecompositores orgânicos desenvolvidos pela Empresa de Pesquisa Agropecuária e Extensão Rural de Santa Catarina - EPAGRI, o processo de degradação ocorre basicamente de forma anaeróbica. Nesse caso, a degradação ocorre dando origem ao biogás (constituído aproximadamente por 60\% de $\mathrm{CH}_{4}$ e $40 \%$ de $\mathrm{CO}_{2}$ ) (NETO, 2007).

O biogás produzido no biodecompositor é expelido com auxílio de um cano que é colocado na parte de cima do dispositivo. Na parte inferior do biodecompositor é adicionada uma torneira para possibilitar a coleta do chorume a cada 10 dias, aproximadamente. Esse líquido, rico em diversas substâncias vitais para as plantas, trata-se de um fertilizante e biopesticida, podendo ser usado para a irrigação de jardins, árvores, plantas e hortaliças (NETO, 2007). Desse modo, o presente projeto tem como principal objetivo confeccionar e instalar seis biodecompositores orgânicos em escolas públicas do município de Garopaba e um biodecompositor no Câmpus Garopaba do IFSC.

\section{MATERIAIS E MÉTODOS}

\footnotetext{
Os biodecompositores orgânicos foram construídos com pouco investimento, a partir de tambores plásticos de 220 litros, usados anteriormente para o armazenamento de azeitonas, conforme Ilustração 1 Biodecompositor orgânico caseiro.

Fonte: Elaborado pelos autores, 2015.
}

\begin{tabular}{|c|c|}
\hline Nome da escola & Bairro de localização \\
\hline Escola Municipal do Pinguirito & Pinguirito \\
\hline $\begin{array}{c}\text { Escola Municipal Professora Jandira Luiza da } \\
\text { Silva }\end{array}$ & Palhocinha \\
\hline Centro Educacional de Ibiraquera & Ibiraquera \\
\hline
\end{tabular}


Confecção e instalação de biodecompositores orgânicos em escolas de educação básica de Garopaba - SC

\begin{tabular}{|c|c|}
\hline Escola Municipal do Macacú & Macacú \\
\hline Escola Municipal Constância Lopes Pereira & Gamboa \\
\hline Escola Municipal Areias de Palhocinha & Areias de Palhocinha \\
\hline Câmpus Garopaba - IFSC & Campo D'una \\
\hline
\end{tabular}

Quadro 1 - Escolas contempladas com o recebimento do biodecompositor orgânico. Fonte: Elaborado pelos autores, 2015.

Em cada escola foi realizada uma capacitação com os servidores responsáveis pelo abastecimento e manipulação dos biodecompositores. Uma cartilha explicativa foi elaborada especificamente para estes servidores fornecendo orientações sobre a correta retirada do chorume, como fazer seu armazenamento e uso. Orientações sobre a manipulação do composto também foram fornecidas, porém, procurou-se dar maior enfoque ao chorume, pois este é produzido em quantidades muito superiores.

As oficinas para a construção dos biodecompositores foram realizadas em conjunto com os extensionistas da EPAGRI conforme a Erro! Autoreferência de indicador não válida..

(a)

(b)

Ilustração 2 - Oficina de construção do biodecompositor com os extensionistas da Epagri Noeli Catarina Pavetto e Anderson Kangerski realizadas na (a) Escola Municipal do Pinguirito e (b) Centro Educacional de Ibiraquera.

Fonte: Elaborado pelos autores, 2015.

Além da realização da oficina para a construção dos biodecompositores envolvendo professores, alunos e demais servidores das escolas, também foram realizadas palestras para conscientização do descarte correto dos resíduos orgânicos, com distribuição de cartilhas educativas e mostra de experimentos para facilitar a compreensão dos alunos em relação aos processos biológicos de decomposição, conforme Ilustração 1 - Biodecompositor orgânico caseiro..

As palestras foram realizadas com experimentos para ilustrar os processos biológicos que ocorrem dentro dos biodecompositores orgânicos e como método de sensibilização sobre as questões ambientais. Para isso, foconfeccionado um minhocário com o uso de baldes de margarina doados por um restaurante de Garopaba e garrafas PET contendo resíduos orgânicos em diferentes estágios de decomposição.

A participação da coordenadora do projeto em reuniões pedagógicas destas escolas também foi realizada visando motivar o uso deste dispositivo como ferramenta pedagógica para as aulas de ciências e matemática por parte dos professores. 
Confecção e instalação de biodecompositores orgânicos em escolas de educação básica de Garopaba - SC

(a)

Ilustração 3 - Realização de palestras com a coordenadora do projeto Sabrina Moro Villela Pacheco e o aluno extensionista Charles Veleda Broqua na (a) Escola Municipal Professora Jandira Luiza da Silva e (b) Escola Municipal do Macacú.

Fonte: Elaborado pelos autores, 2015.

\section{RESULTADOS E ANÁLISES}

Com a implantação dos biodecompositores orgânicos nas escolas básicas Ilustração 1 Biodecompositor orgânico caseiro., pretendeu-se, além da conscientização ambiental para o descarte adequado dos resíduos, estimular, nas escolas da região, a criação de hortas orgânicas. Tal estímulo foi realizado devido ao fato do biodecompositor produzir grandes quantidades de líquidos que são obtidos no processo de biodegradação e podem ser usados como fertilizante em jardins e gramados, assim como na irrigação de hortaliças. Outro aspecto muito interessante do líquido produzido é que o mesmo também pode ser utilizado como biopesticida, auxiliando no processo de obtenção dos alimentos orgânicos. Desse modo, além da sensibilização provocada para questões do lixo, o projeto também possui relação direta com o incentivo a uma alimentação mais saudável. A parceria IFSC e EPAGRI foi muito produtiva, pois as escolas também receberam diversas sementes para a implantação da horta.

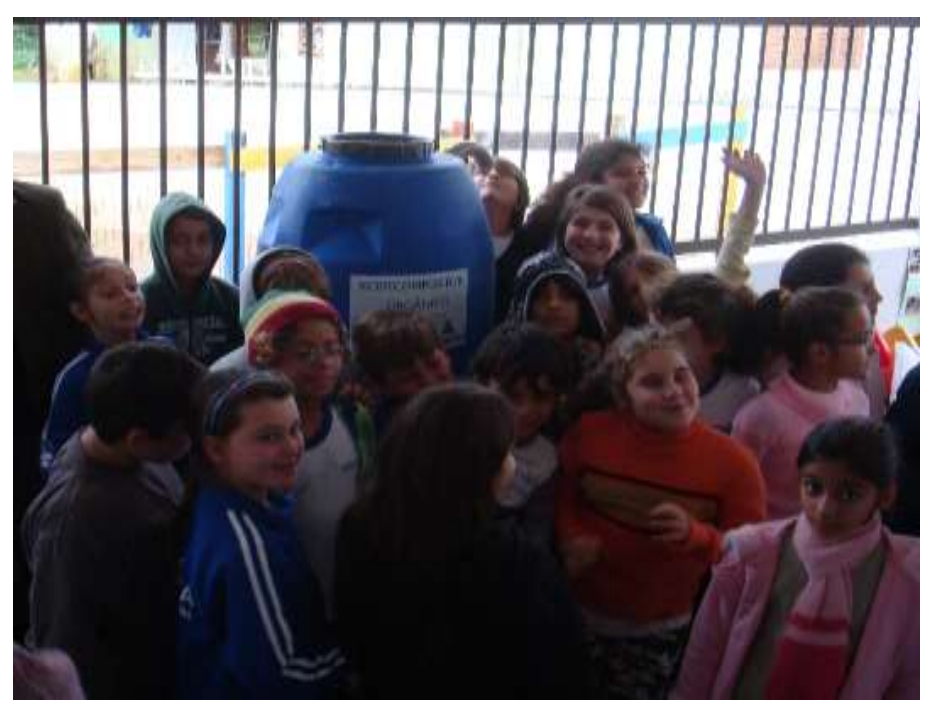

Ilustração 4. Para a construção dos biodecompositores foram usados os materiais listados no Quadro 1. 
Confecção e instalação de biodecompositores orgânicos em escolas de educação básica de Garopaba - SC

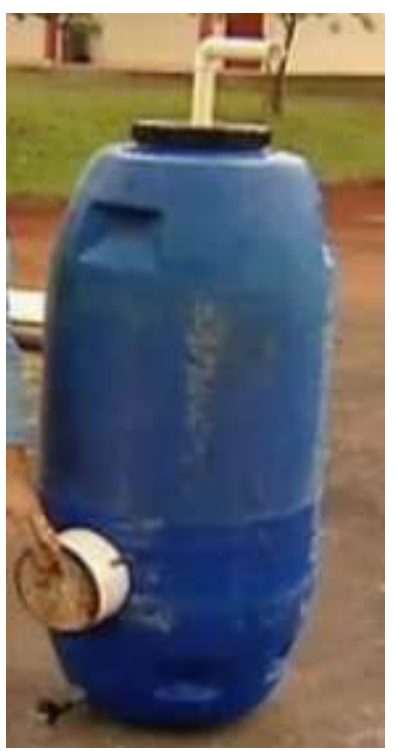

Ilustração 1 - Biodecompositor orgânico caseiro. Fonte: Elaborado pelos autores, 2015.

\begin{tabular}{|c|c|}
\hline Nome da escola & Bairro de localização \\
\hline Escola Municipal do Pinguirito & Pinguirito \\
\hline $\begin{array}{c}\text { Escola Municipal Professora Jandira Luiza da } \\
\text { Silva }\end{array}$ & Palhocinha \\
\hline Centro Educacional de Ibiraquera & Ibiraquera \\
\hline Escola Municipal do Macacú & Macacú \\
\hline Escola Municipal Constância Lopes Pereira & Gamboa \\
\hline Escola Municipal Areias de Palhocinha & Areias de Palhocinha \\
\hline Câmpus Garopaba - IFSC & Campo D'una \\
\hline
\end{tabular}

Quadro 1 - Escolas contempladas com o recebimento do biodecompositor orgânico. Fonte: Elaborado pelos autores, 2015.

Em cada escola foi realizada uma capacitação com os servidores responsáveis pelo abastecimento e manipulação dos biodecompositores. Uma cartilha explicativa foi elaborada especificamente para estes servidores fornecendo orientações sobre a correta retirada do chorume, como fazer seu armazenamento e uso. Orientações sobre a manipulação do composto também foram fornecidas, porém, procurou-se dar maior enfoque ao chorume, pois este é produzido em quantidades muito superiores.

As oficinas para a construção dos biodecompositores foram realizadas em conjunto com os extensionistas da EPAGRI conforme a Erro! Autoreferência de indicador não válida.. 
Confecção e instalação de biodecompositores orgânicos em escolas de educação básica de Garopaba - SC

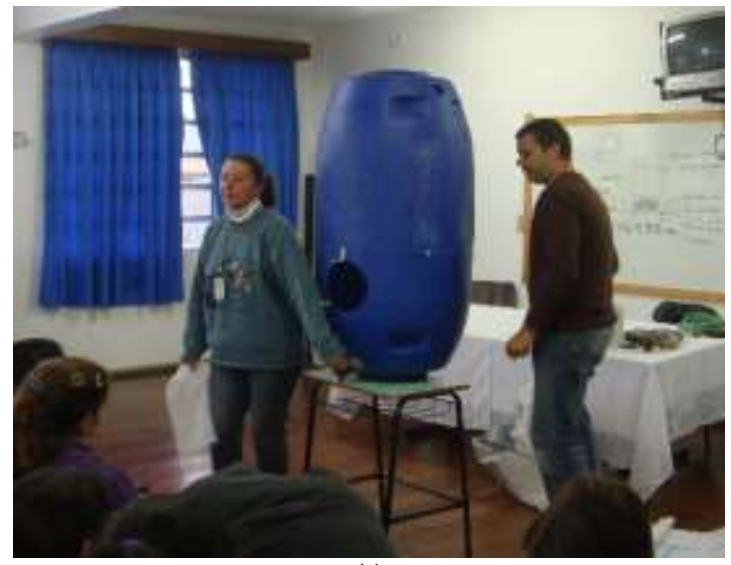

(a)

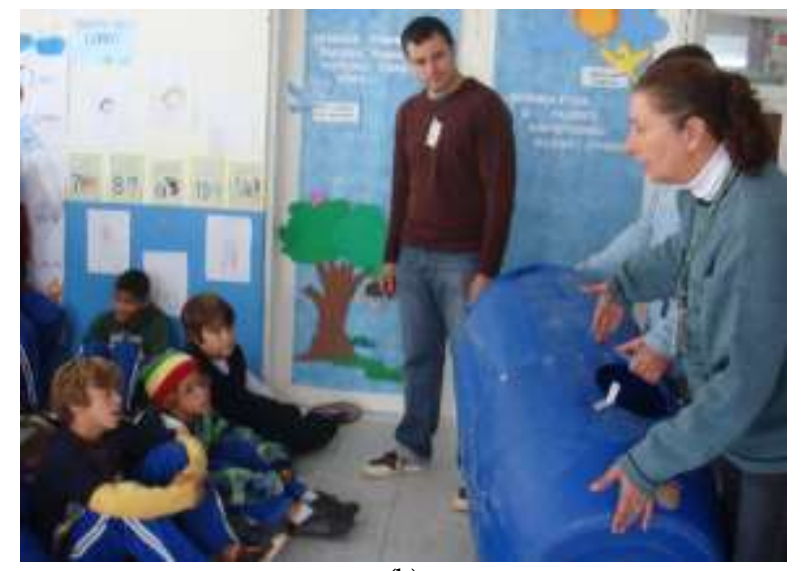

(b)

Ilustração 2 - Oficina de construção do biodecompositor com os extensionistas da Epagri Noeli Catarina Pavetto e Anderson Kangerski realizadas na (a) Escola Municipal do Pinguirito e (b) Centro Educacional de Ibiraquera. Fonte: Elaborado pelos autores, 2015.

Além da realização da oficina para a construção dos biodecompositores envolvendo professores, alunos e demais servidores das escolas, também foram realizadas palestras para conscientização do descarte correto dos resíduos orgânicos, com distribuição de cartilhas educativas e mostra de experimentos para facilitar a compreensão dos alunos em relação aos processos biológicos de decomposição, conforme 0Erro! Autoreferência de indicador não válida..

As palestras foram realizadas com experimentos para ilustrar os processos biológicos que ocorrem dentro dos biodecompositores orgânicos e como método de sensibilização sobre as questões ambientais. Para isso, foconfeccionado um minhocário com o uso de baldes de margarina doados por um restaurante de Garopaba e garrafas PET contendo resíduos orgânicos em diferentes estágios de decomposição.

A participação da coordenadora do projeto em reuniões pedagógicas destas escolas também foi realizada visando motivar o uso deste dispositivo como ferramenta pedagógica para as aulas de ciências e matemática por parte dos professores. 
Confecção e instalação de biodecompositores orgânicos em escolas de educação básica de Garopaba - SC

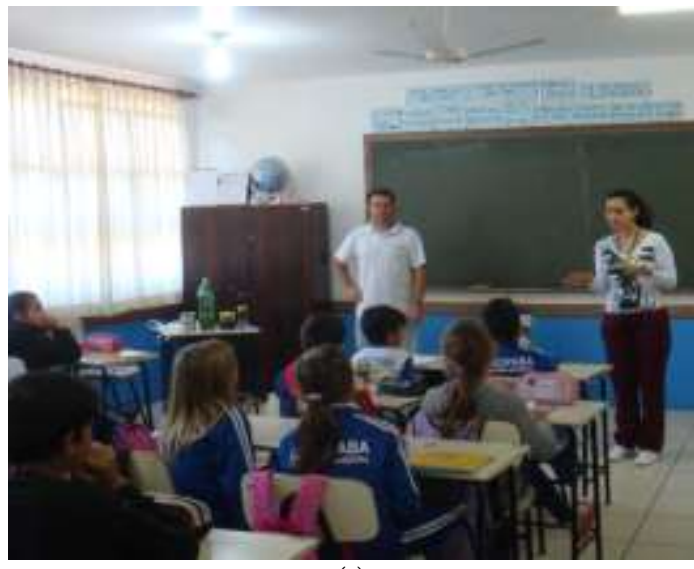

(a)

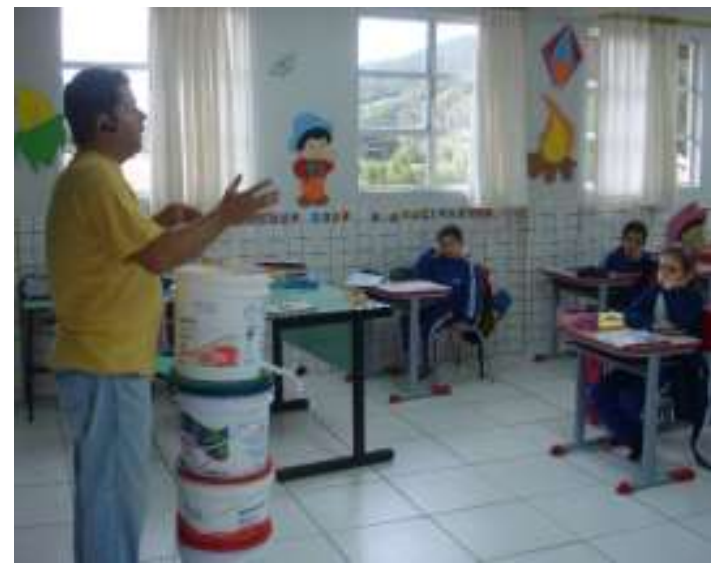

(b)

Ilustração 3 - Realização de palestras com a coordenadora do projeto Sabrina Moro Villela Pacheco e o aluno extensionista Charles Veleda Broqua na (a) Escola Municipal Professora Jandira Luiza da Silva e (b) Escola Municipal do Macacú.

Fonte: Elaborado pelos autores, 2015.

\section{RESULTADOS E ANÁLISES}

Com a implantação dos biodecompositores orgânicos nas escolas básicas Erro! Autoreferência de indicador não válida., pretendeu-se, além da conscientização ambiental para o descarte adequado dos resíduos, estimular, nas escolas da região, a criação de hortas orgânicas. Tal estímulo foi realizado devido ao fato do biodecompositor produzir grandes quantidades de líquidos que são obtidos no processo de biodegradação e podem ser usados como fertilizante em jardins e gramados, assim como na irrigação de hortaliças. Outro aspecto muito interessante do líquido produzido é que o mesmo também pode ser utilizado como biopesticida, auxiliando no processo de obtenção dos alimentos orgânicos. Desse modo, além da sensibilização provocada para questões do lixo, o projeto também possui relação direta com o incentivo a uma alimentação mais saudável. A parceria IFSC e EPAGRI foi muito produtiva, pois as escolas também receberam diversas sementes para a implantação da horta. 
Confecção e instalação de biodecompositores orgânicos em escolas de educação básica de Garopaba - SC

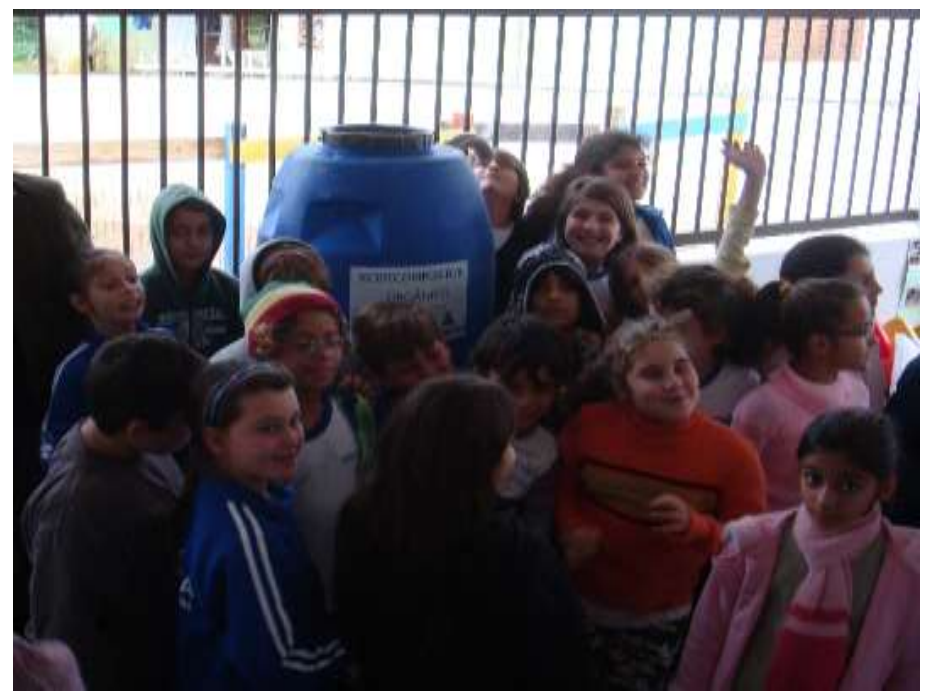

Ilustração 4 - Biodecompositor orgânico sendo instalado no Centro Educacional de Ibiraquera.

Fonte: Elaborado pelos autores, 2015.

O grande volume de chorume gerado pelo biodecompositor apresentou aspectos positivos e negativos. O aspecto negativo observado por algumas escolas foi o fato das mesmas não conseguirem utilizar todo o chorume produzido . Especialmente as escolas que apresentavam um número de alunos superior a cem crianças, pois geram maior volume de líquidos provenientes do processo de biodecomposição . Este fato pode ser contornado pelas inúmeras aplicações que o chorume pode ter. Neste sentido, é possível citar o exemplo da Escola Municipal Professora Jandira Luiza da Silva que utilizou o mesmo com sucesso para desentupir a pia da cozinha. Existem diversos relatos na internet citando a possibilidade desta aplicação, porém, tal uso ainda não havia sido testado pela EPAGRI e o IFSC. É importante ressaltar que possivelmente o sucesso do processo só foi possível devido ao fato de ter sido utilizado o chorume coletado no mesmo dia do biodecompositor.

Após a finalização do projeto, diversas pessoas fora da comunidade escolar entraram em contato, procurando saber mais sobre como construir o dispositivo. Dentre os diversos contatos realizados, é possível destacar o interesse de pequenos agricultores e donos de meios de hospedagem para a instalação do dispositivo. Tais eventos ilustram a variedade das futuras parcerias para a realização de outros projetos. O fato dos pais receberem as informações de seus filhos - alunos das escolas - e a facilidade do uso do dispositivo são apenas alguns dos reflexos positivos que o projeto gerou até o momento. 
Confecção e instalação de biodecompositores orgânicos em escolas de educação básica de Garopaba - SC

\section{CONSIDERAÇÕES FINAIS}

Através dos resultados obtidos, foi possível verificar que o projeto cumpriu com seu objetivo principal de reduzir a quantidade de resíduos gerados pelo município e adotar ações de reciclagem. As escolas foram escolhidas para a implantação do biodecompositores por serem espaços de reflexão, educação, conscientização e principalmente, de motivação para a adoção de ações de sustentabilidade pelas crianças de Garopaba. É possível dizer que, ao todo foram atingidas 1000 pessoas que deverão auxiliar como multiplicadores nas informações trabalhadas.

O projeto apresentou pontos negativos, dentre os quais é possível citar:

a) Reunião com diretores antes da instalação do biodecompositor. Este é um aspecto importante, pois a decisão de quais escolas seria contemplada foi tomada pela Prefeitura Municipal de Garopaba. Infelizmente, nem todas as escolas possuíam condições físicas e de pessoal para auxiliar na instalação e até mesmo no manuseio do dispositivo. Esse fato está diretamente associado ao segundo ponto negativo;

b) Falta de pessoal para o manuseio do biodecompositor. De modo geral, o manuseio do dispositivo foi realizado pelas merendeiras ou serventes. O manuseio foi realizado por uma professora e sua turma de alunos, apenas no Centro Educacional de Ibiraquera. Este fato é preocupante, pois existe a necessidade de retirada semanal de chorume. Se esta medida não é adotada, podem ocorrer vazamentos, mau cheiro e proliferação de insetos;

c) O terceiro aspecto negativo foi a tampa de madeira adicionada aos biodecompositores. Em alguns casos a tampa sofreu ruptura ou inchaço devido ao encharcamento da madeira com água da chuva, mesmo sendo revestida com um saco plástico. Optou-se por essa alternativa devido ao elevado custo de uma tampa de PVC para o cano de $200 \mathrm{~mm}$, porém, esta ainda sim é a melhor opção;

d) Pouco uso didático dos biodecompositores por parte da equipe pedagógica da maior parte das escolas. Nesse caso, infelizmente os dispositivos foram utilizados como ferramenta para o ensino de ciências e matemática apenas no Centro Educacional de Ibiraquera. Esse fato é considerado como negativo, já que algumas reuniões foram realizadas com o corpo docente da maior parte das escolas, para fomentar e incentivar o uso do biodecompositor para este fim. Inúmeros são os conceitos possivelmente abordados com o uso dos biodecompositores como temas geradores, especialmente para o ensino de ciências e matemática.

Em relação aos aspectos positivos é possível destacar: 
Confecção e instalação de biodecompositores orgânicos em escolas de educação básica de Garopaba - SC

a) A receptividade e o interesse dos alunos pelo tema. Conforme citado anteriormente, antes da implantação dos biodecompositores, palestras informativas foram realizadas nas escolas, procurando-se abranger o maior número de turmas possíveis. Através desse contato, foi possível observar grande interesse e motivação por parte dos alunos para participar do projeto. Alguns alunos inclusive comentaram que iriam levar a ideia para seus pais com o objetivo de implementarem o dispositivo em suas próprias casas;

b) A implantação do projeto nas escolas abrangeu um público maior e mais diversificado que o esperado. Esse fato deve ser explicado possivelmente pelo fato dos dispositivos ficarem em locais de fácil observação por parte dos pais e outros visitantes das escolas. Isso ocorreu, pois os dispositivos necessitam que muita luz solar, o que inviabiliza seu uso em locais fechados;

c) Necessidade e possibilidade de realização de outros projetos. Foi possível perceber que o acompanhamento de um biodecompositor é um passo tão importante quanto à sua instalação. É necessário verificar o manuseio, fazer reparos e ofertar capacitação constante. Esse fato é destacado como positivo, pois abre portas para a continuidade do projeto.

Além disso, existem outras possibilidades de realização deste projeto com meios de hospedagem e restaurantes do município. Esse fato possui relação com os aspectos econômicos, pois é importante citar que a diminuição do lixo como um todo é atraente para as prefeituras. Atualmente maior parte do lixo do município de Garopaba é enviada ao aterro sanitário de Biguaçu através de caminhões. Esse deslocamento é realizado com o uso de recursos financeiros, que, por sua vez, podem ser reduzidos concomitantemente à redução do lixo.

\section{REFERÊNCIAS}

ASSOCIAÇÃO BRASILEIRA DE NORMAS TÉCNICAS. NBR 10004: Resíduos sólidos Classificação. Rio de Janeiro, 2004. 71 p.

BIDONE, Francisco Ricardo Andrade; POVINELLI, Jurandyr. 1999. Conceitos Básicos de Resíduos Sólidos. São Carlos: EESC/USP.

IBGE, Instituto Brasileiro de Geografia e Estatística. IBGE Cidades. Disponível em: <http://www.ibge.gov.br/home/presidencia/noticias/noticia_visualiza.php?id_noticia=1998\&id _pagina=1>. Acesso em: 27 fev. 2013. 
Confecção e instalação de biodecompositores orgânicos em escolas de educação básica de Garopaba - SC

IBGE Cidades. Disponível em: <http://www.ibge.gov.br/cidadesat/topwindow.htm?1>. Acesso em: 27 fev. 2013.

INÁCIO, Caio de Teves; MILLER, Paul Richard Monsem. 2009. Compostagem - Ciência e Prática para a Gestão de Resíduos Orgânicos. Rio de Janeiro: Embrapa Solos.

IPEA, Instituto de Pesquisa Econômica Aplicada. Diagnósticos dos Resíduos Sólidos Urbanos.

<http://www.ipea.gov.br/agencia/images/stories/PDFs/relatoriopesquisa/121009_relatorio_re siduos_solidos_urbanos.pdf $>$. Acesso em: 25 fev. 2014.

JARDIM, N.S.; WELLS. C.; CONSONI. A.J.; AZEVEDO. R.M.B. 2000. Gerenciamento integrado do lixo municipal. In: D'ALMEIDA. M.L.O.; VILHENA. A. (Org). Lixo municipal: manualde gerenciamento integrado. São Paulo: IPT/CEMPRE.

NETO, João Tinôco Pereira. 2007. Manual de Compostagem - processo de baixo custo. Belo Horizonte: UFV. 